\title{
Articles
}

\section{Synthesis of Thia(oxa)zolopyridines and Their Inhibitory Activities for $\beta$-Amyloid Fibrillization}

\author{
Yeo Ran Lee, Dong Jin Kim. Inhee Mook-Jung. ${ }^{\dagger}$ and Kyung Ho Yoo \\ Life Sciences Research Division, Korea Institute of Science and Technology, P.O. Box 131, Cheongryang, Seoul 130-650, Korea \\ "E-mail.khyoo@kist.re.k. \\ "Department of Biochemistry and Cancer Research Institute, Seoul National University College of Medicine, Seoul 110-799, Korea \\ Received August 15, 2008
}

\begin{abstract}
A series of thiazolo $[5,4-b]$ pydine and oxazolo $[5,4-b]$ pyridine derivatives 1a-o were designed. These fifteen compounds were evaluated by $\mathrm{A} \beta 42$ fibril formation inhibitory assay using thioflavin $\mathrm{T}$ as a dye. Thiazolo[5,4$b$ ]pyridines 1a-g showed potent inhibitory activities for $\mathrm{A} \beta 42$ fibrillization at $\mathrm{IC}_{50}$ of $0.23-4.5 \mu \mathrm{M}$. Among them, compounds $1 \mathbf{b}$ and $1 \mathbf{k}$ having methoxy group at $\mathrm{C}-5$ exhibited excellent activities $\left(\mathrm{IC}_{50}=0.23\right.$ and 0.50 $\mu \mathrm{M}$, respectively) than that of Curcumin ( $\mathrm{IC}_{s 0}=0.80 \mu \mathrm{M}$ ).
\end{abstract}

Key Words : $\Lambda$ lzheimer's disease, is $\Lambda$ myloid fibrillization. Thia(oxa)zolopyridines, Binding affinity

\section{Introduction}

Alzheimer's disease (AD) is a neurodegenerative disorder that is the most common cause of dementia among elderly people.' Fibrillar amyloid- $\beta$ peptide $(\mathrm{A} \beta)$, a pathological hallmark of $\mathrm{AD}_{3}{ }^{2-4}$ is one of the main components of senile plaques. Formation and accumulation of aggregates of $\mathrm{A} \beta$ peptides in the brain are critical factors in the onset of $\mathrm{AD}{ }^{5.6}$ Two major $\mathrm{A} \beta$ peptides $\mathrm{A} \beta 40$ and $\mathrm{A} \beta 42$ of different lengths have been derived from amyloid precursor protein (APP) ${ }^{7.8}$ Fibrillization of $A \beta 42$ occurs via oligomers and protofibrils more readily than relatively soluble $A \beta 40.9 .10$

Preventing and reversal of $\mathrm{A} \beta$ fibril formation in the brain are currently being targeted as a potential therapeutic approach to $\mathrm{AD} \cdot{ }^{1-1 / 3}$ Recently, several series of inhibitors such as $E, E$-1-iodo-2,5-bis-(3-hydroxycarbonyl-4-methoxy)styrylbenzene (IMSB), ${ }^{14,15}$ (IE,6E)-1,7-bis(4-hydroxy-3methoxyphenyl)-1,6-heptadiene-3,5-dione (Curcumin), ${ }^{16.17}$ 4- $\mathrm{N}$-methylamino-4'-hydroxystilbene (SB-13), ${ }^{18}$ and benzofuran analogues ${ }^{19}$ have been described, and these compounds were found to inhibit effectively $\mathrm{A} \beta$ fibril formation as determined by thioflavin $\mathrm{T}$ (ThT) assay. In addition, $N$ methyl-[" $\mathrm{C}] 2-\left(4^{-}-\right.$-methylaminopheny] $)-6$-hydroxybenzothiazole $\left.\left({ }^{11} \mathrm{C}\right] \mathrm{PIB}\right)^{2021}$ exhibited high affinity for $A \beta$, good brain entry and clearance.

In an effort to develop a treatment for $\mathrm{AD}$, the structural modification by the introduction of thia(oxa)zolopyridine with an additional binding site instead of benzothiazole nucleus of PIB was designed as shown in Figure 1 . We report here the synthesis and evaluation of a series of thia(oxa)zolo[5,4-b]pyridine derivatives 1:-o with potent inhibitory activities for $\mathrm{A} \beta$ fibrillization.

\section{Results and Discussion}

Chemistry. Thiazolo[5,4-b]pyridines 1a-g and oxazolo$[5,4-b]$ pyridines 1l-o with $p-N, N$-dimethylaminophenyl group were prepared by the sequence outlined in Scheme 1 .

Reduction of nitro group of 3-nitropyridines $2 \mathbf{a}, \mathbf{b}$ by tin chloride ${ }^{22}$ gave the amino compounds $3 \mathbf{a}, \mathbf{b}$ and subsequent amidation of 3 (3c-g: commercially available) with 4-dimethylaminobenzoyl chloride in pyridine afforded the corresponding benzamides $4 \mathrm{a}-\mathrm{f}$, respectively. Conversion of carbonyl group of 4a-f to thiocarbonyl group with Lawesson's reagent, followed by ring-closure provided the desired thiazolo $[5,4-b]$ pyridines 1a-g. ${ }^{23}$ Treatment of $\mathbf{4}$ with phosphorus pentoxide (for $11, \mathrm{~m}$ ) or polyphosphoric acid (for 1n, $\mathbf{o})$ resulted in the corresponding oxazolo $[5,4-b]$ pyridines 11-o, respectively. ${ }^{24}$ Hydroxyl compound 1c was obtained from methoxy compound $\mathbf{1 b}$ by demethylation using boron tribromide. $^{25}$

The synthesis of thiazolo[5,4-b]pyridines $1 \mathbf{h}-\mathbf{k}$ with $p$ -

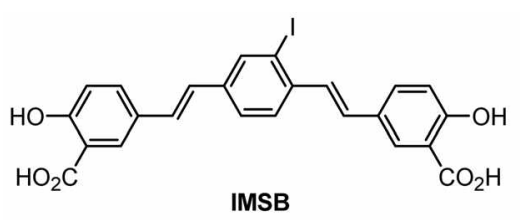

IMSB

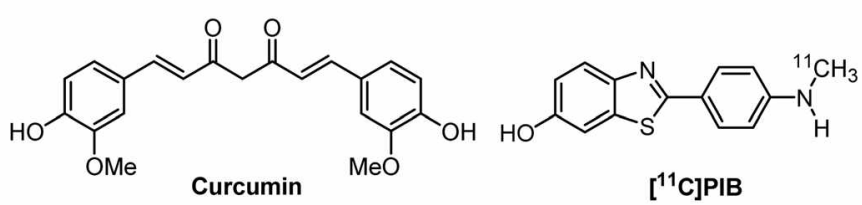

$\left[{ }^{11} \mathrm{C}\right] \mathrm{PIB}$

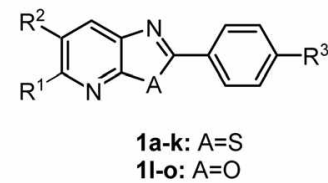

1a-k: $A=S$
11-o: $A=0$

Figure 1. Structures of IMSB, Curcumin, [ $\left.{ }^{11} \mathrm{C}\right] \mathrm{PIB}$, and Thia(Oxa)zolopyridines. 


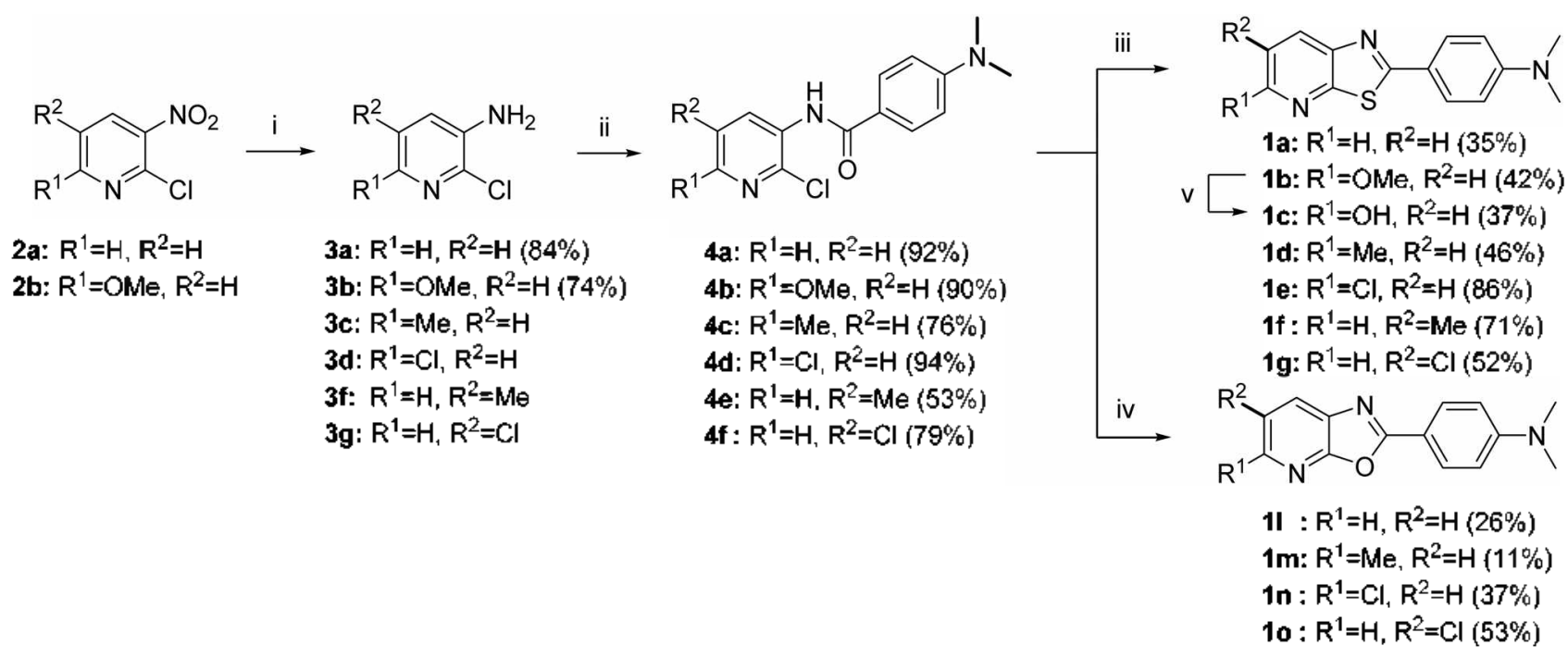

Scheme 1. Reagents and reaction conditions: (i) $\mathrm{SnCl}$, $\mathrm{EtOH}$, reflux, $30 \mathrm{~min}-2 \mathrm{~h}$; (ii) 4-dinethylaminobenzoyl chloride, pyridine, $\mathrm{rt}, 18 \mathrm{~h}$; (iii) Lawesson's reagent, chlorobenzene, reflux, $3 \mathrm{~h}$; (iv) $\mathrm{P} \div \mathrm{O}_{3}$, hexamethyldisiloxane, 1,2-dichlorobenzene, $140{ }^{\circ} \mathrm{C}, 2 \mathrm{~d}$ (for $1 \mathrm{l}, \mathrm{m}$ ) or polyphosphoric acid, dichloromethane, $140^{\circ} \mathrm{C}, 1 \mathrm{~d}$ (for $1 \mathrm{n}, 0$ ) (v) $\mathrm{BBr}_{3}$, dichloromethane, reflux, $12 \mathrm{~h}$.
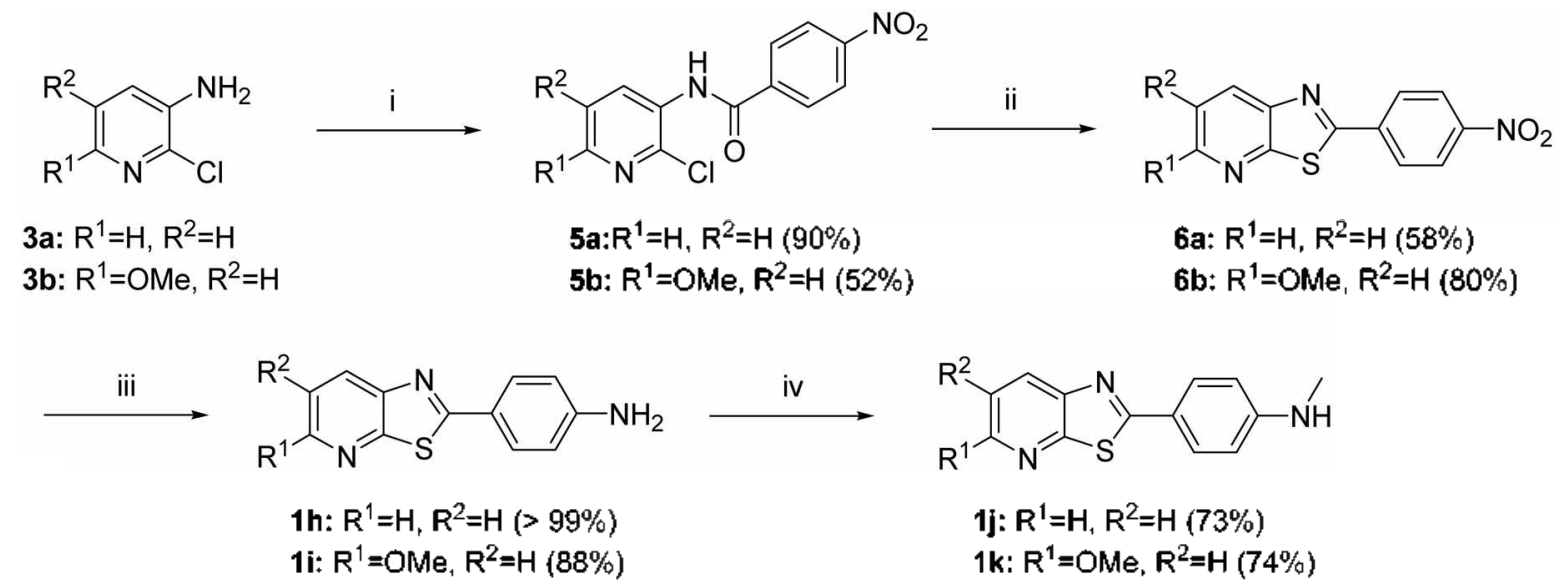

Scheme 2. Reagents and reaction conditions: (i) 4-nitrobenzoyl chloride, pyridinc, $\mathrm{rt}, 18 \mathrm{~h}$; (ii) Lawesson's Reagent, chlorobenzene, reflux, $3 \mathrm{~h}$; (iii) $\mathrm{SnCl}_{2}$, EtOH, reflux, 6-18 hr (iv) $\left(\mathrm{CH}_{2} \mathrm{O}\right) \mathrm{n}$, $\mathrm{NaOMe}, \mathrm{MeOH}, \mathrm{NaBH}_{4}$, reflux, $5 \mathrm{~h}$.

aminophenyl or $p$ - $N$-methylaminophenyl group was outlined in Scheme 2.

Amidation of amino compounds $\mathbf{3 a}, \mathbf{b}$ and ring-closure of the resulting amides $\mathbf{5 a}, \mathbf{b}$ were carried out by using procedures analogous to those described above to provide $1 \mathrm{a}-\mathrm{g}$. Reduction of nitro group of $6 \mathbf{a}, \mathbf{b}$ by tin chloride led to the amino compounds $\mathbf{1 h}, \mathbf{i}$, which were methylated with paraformaldehyde and sodium borohydride in the presence of sodium methoxide to yield the terminal secondary amines 1j. . $^{3+}$

Biological evaluation. Table 1 shows the in vitro inhibjtory activities ( $\mathrm{IC}_{50}$ values) of thia(oxa)zolo[5,4-b]pyridines 1a-0 for $A \beta 42$ fibril formation together with those of Curcumin and IMSB as reference compounds.

All the synthesized compounds were evaluated by thioflavin $\mathrm{T}$ (ThT) assay ${ }^{27}$ as shown in Table 1, twelve compounds showed very good inhibitory activities with $\mathrm{IC}_{50}$ of 0.23-4.5 $\mu \mathrm{M}$. As a whole, thiazolo[5,4-b]pyridines 1a-k displayed better potency for $\mathrm{A} \beta 42$ fibril formation than oxazolo[5,4-b]pyridines 11-o. Especially, compounds 1b and $1 \mathrm{k}$ with methoxy group at $\mathrm{C}-5$ exhibited excellent inhibitory activities ( $\mathrm{IC}_{50}=0.23$ and $0.50 \mu \mathrm{M}$, respectively) compared to Curcumin $\left(\mathrm{IC}_{50}=0.80 \mu \mathrm{M}\right)$. Changing methoxy group of 1b to hydroxyl group of $1 \mathrm{c}$ markedly decreased the activity. In the structure-activity relationship, positional and electronic effects according to $\mathrm{R}^{1}$ and $\mathrm{R}^{2}$ substituents did not make any significant difference of inhibitory activity. The variation of amine substituents $\mathrm{R}^{3}$ did not show a meaningful trend.

In conclusion, a series of thiazolo[5,4-b]pyridines based on the structural modification of PIB showed potent inhibitory activities for $\beta$-amyloid fibrillization. In particular, compounds $1 \mathbf{b}$ and $1 \mathbf{k}$ exhibited superior inhibitory activities to Curcumin. This preliminary result suggests that these compounds possess a possibility as a treatment for $\mathrm{AD}$. 
Table 1. Inhibitory activitics of thia(oxa)zolopyridines $1 a-0$ for $\beta$ amyloid fibrillization

\begin{tabular}{lccccc}
\hline Compds & $\mathrm{A}$ & $\mathrm{R}_{1}$ & $\mathrm{R}_{2}$ & $\mathrm{R}_{3}$ & $\mathrm{IC}_{50}\left(u \mathrm{M}^{a}\right.$ \\
\hline $\mathbf{l a}$ & $\mathrm{S}$ & $\mathrm{H}$ & $\mathrm{H}$ & $\mathrm{NMe}_{2}$ & 2.7 \\
$\mathbf{1 b}$ & $\mathrm{S}$ & $\mathrm{MeO}$ & $\mathrm{H}$ & $\mathrm{NMe}_{2}$ & 0.23 \\
$\mathbf{1 c}$ & $\mathrm{S}$ & $\mathrm{HO}$ & $\mathrm{H}$ & $\mathrm{NMe}_{2}$ & 2.8 \\
$\mathbf{1 d}$ & $\mathrm{S}$ & $\mathrm{Me}$ & $\mathrm{H}$ & $\mathrm{NMe}_{2}$ & 2.6 \\
$\mathbf{l e}$ & $\mathrm{S}$ & $\mathrm{Cl}$ & $\mathrm{H}$ & $\mathrm{NMe}_{2}$ & 1.1 \\
$\mathbf{1 f}$ & $\mathrm{S}$ & $\mathrm{H}$ & $\mathrm{Me}$ & $\mathrm{NMe}_{2}$ & 2.6 \\
$\mathbf{l g}$ & $\mathrm{S}$ & $\mathrm{H}$ & $\mathrm{Cl}$ & $\mathrm{NMe}_{2}$ & 4.3 \\
$\mathbf{1 h}$ & $\mathrm{S}$ & $\mathrm{H}$ & $\mathrm{H}$ & $\mathrm{NH}_{2}$ & 1.5 \\
$\mathbf{l i}$ & $\mathrm{S}$ & $\mathrm{MeO}$ & $\mathrm{H}$ & $\mathrm{NH}_{2}$ & 4.5 \\
$\mathbf{1 j}$ & $\mathrm{S}$ & $\mathrm{H}$ & $\mathrm{H}$ & $\mathrm{NHMe}^{2}$ & 1.7 \\
$\mathbf{1 k}$ & $\mathrm{S}$ & $\mathrm{MeO}$ & $\mathrm{H}$ & $\mathrm{NHMe}^{2}$ & 0.50 \\
$\mathbf{1 l}$ & $\mathrm{O}$ & $\mathrm{H}$ & $\mathrm{H}$ & $\mathrm{NMe}_{2}$ & 16 \\
$\mathbf{1 m}$ & $\mathrm{O}$ & $\mathrm{Me}$ & $\mathrm{H}$ & $\mathrm{NMe}_{2}$ & 3.5 \\
$\mathbf{1 n}$ & $\mathrm{O}$ & $\mathrm{Cl}$ & $\mathrm{H}$ & $\mathrm{NMe}_{2}$ & 11 \\
$\mathbf{l o}$ & $\mathrm{O}$ & $\mathrm{H}$ & $\mathrm{Cl}$ & $\mathrm{NMe}_{2}$ & 15 \\
Curcumin & & & & & 0.80 \\
IMSB & & & & & 8.00 \\
\hline
\end{tabular}

"ThT assay. $\mathrm{IC}_{\text {so }}$ was calculated from non-linear redression by Grop/pad Prisns soft-ware.

\section{Experimental}

Melting points were measured with a Thomas Hoover capillary melting point apparatus and were uncorrected. IR spectra were obtained on a Perkin-Elmer FT-IR system spectrophotometer. 'H NMR and ${ }^{13} \mathrm{C} \mathrm{NMR}$ spectra were recorded on a Bruker Avance 300 spectrometer $(300 \mathrm{MHz}$ for ${ }^{1} \mathrm{H}$ and $75 \mathrm{MHz}$ for ${ }^{13} \mathrm{C}$ ) using tetramethylsilane as an internal standard. LC-Mass spectra were determined on a Waters Quattro Micro system. Column chromatography was carried out using silica gel (230-400 mesh). Solvents and liquid reagents were transferred using hypodermic syringes. All other reagents and solvents used were reagent grade.

General procedure for the synthesis of 2-chloropyridin-3-ylamines 3a,b. To a solution of the appropriate 3nitropyridine compound $2(2.0 \mathrm{mmol})$ in ethanol $(15 \mathrm{~mL})$ was added tin chloride $(10.0 \mathrm{mmol})$, and the reaction mixture was refluxed for $30 \mathrm{~min}-2 \mathrm{~h}$. When the reaction was completed, the reaction mixture was treated with $10 \%$ $\mathrm{NaHCO}_{3}$. The mixture was extracted with ethyl acetate, dried over anhydrous $\mathrm{Na}_{2} \mathrm{SO}_{4}$, and concentrated in vacuo. The residue was purified by silica gel column chromatography to give the compound 3 .

2-Chloropyridin-3-ylamine (3a). Yield 84\%; white solid; 'H NMR ( $\left.300 \mathrm{MHz}, \mathrm{CDCl}_{3}\right) \delta 7.81$ (dd, $1 \mathrm{H}, J=1.9,4.3 \mathrm{~Hz}$ ), 7.10 (dd, $1 \mathrm{H}, J=4.3,7.9 \mathrm{~Hz}), 7.05$ (dd, $1 \mathrm{H}, J=1.9,7.9 \mathrm{~Hz}$ ), 4.11 (br s, 2H).

6-Methoxy-2-chloropyridin-3-ylamine (3b). Yield 74\%; white solid; ${ }^{1} \mathrm{H}$ NMR $\left(300 \mathrm{MHz}, \mathrm{CDCl}_{3}\right) \delta 7.09$ (d, $1 \mathrm{H}, J=$ $8.5 \mathrm{~Hz}), 6.58(\mathrm{~d}, 1 \mathrm{H}, J=8.5 \mathrm{~Hz}), 3.89$ (s, 3H), 3.69 (br s, $2 \mathrm{H}$ ).

General procedure for the synthesis of $N$-(2-chloropyridin-3-yl)-4-dimethylaminobenzamides 4a-f. To a solution of the appropriate 3-aminopyridine compound 3 $(10.0 \mathrm{mmol})$ in pyridine $(5 \mathrm{~mL})$ was added 4-dimethylaminobenzoyl chloride $(10.0 \mathrm{mmol})$ in pyridine $(5 \mathrm{~mL})$, and the reaction mixture was stirred at room temperature for 18 $\mathrm{h}$. When the reaction was completed, the reaction mixture was treated with water. The resulting precipitate was collected by filtration to give the compound 4 .

$\mathrm{N}$-(2-Chloropyridin-3-yl)-4-dimethylaminobenzamide (4a). Yield 92\%; white solid; 'H NMR (300 MHz, $\left.\mathrm{CDCl}_{3}\right) \delta$ 8.93 (dd, $1 \mathrm{H}, J=1.7,8.2 \mathrm{~Hz}$ ), 8.34 (br s, $1 \mathrm{H}$ ), 8.11 (dd, $1 \mathrm{H}$, $J=1.7,4.7 \mathrm{~Hz}), 7.82(\mathrm{~d}, 2 \mathrm{H}, J=9.0 \mathrm{~Hz}), 7.30(\mathrm{dd}, 1 \mathrm{H}, J=$ $4.7,8.2 \mathrm{~Hz}), 6.73(\mathrm{~d}, 2 \mathrm{H}, J=9.0 \mathrm{~Hz}), 3.07(\mathrm{~s}, 6 \mathrm{H}) ;{ }^{13} \mathrm{C}$ NMR (75 $\left.\mathrm{MHz}, \mathrm{CDCl}_{3}\right) \delta 165.5,153.1,143.1,139.8,132.7,128.8$, $128.5,123.5,120.0,111.2,40.1$; MS $m / z 276(\mathrm{M}+\mathrm{H})^{+}$.

6-Methoxy- $N$-(2-chloropyridin-3-yl)-4-dimethylaminobenzamide (4b). Yield 90\%; white solid; ${ }^{1} \mathrm{H}$ NMR $(300$ $\left.\mathrm{MHz}, \mathrm{CDCl}_{3}\right) \delta 8.74(\mathrm{~d}, 1 \mathrm{H}, J=9.0 \mathrm{~Hz}$ ), 8.05 (br s, $1 \mathrm{H}$ ), $7.82(\mathrm{~d}, 2 \mathrm{H}, J=9.0 \mathrm{~Hz}), 6.75(\mathrm{~m}, 3 \mathrm{H}), 3.94(\mathrm{~s}, 3 \mathrm{H}), 3.08(\mathrm{~s}$, $6 \mathrm{H}) ;{ }^{13} \mathrm{C} \mathrm{NMR}\left(75 \mathrm{MHz}, \mathrm{CDCl}_{3}\right) \delta 165.2,159.0,152.8$, $136.5,133.4,128.7,126.1,120.8,111.4,109.8,54.1,40.2$.

6-Methyl- $N$-(2-chloropyridin-3-yl)-4-dimethylaminobenzamide (4c). Yield 76\%; white solid; ${ }^{1} \mathrm{H}$ NMR $(300$ $\left.\mathrm{MHz}_{3} \mathrm{CDCl}_{3}\right) \delta 8.79(\mathrm{~d}, 1 \mathrm{H}, J=8.2 \mathrm{~Hz}$ ), 8.25 (br s, $1 \mathrm{H}$ ), $7.82(\mathrm{~d}, 2 \mathrm{H}, J=9.0 \mathrm{~Hz}), 7.15$ (d, 1H. $J=8.2 \mathrm{~Hz}), 6.74(\mathrm{~d}$, $2 \mathrm{H}, J=9.0 \mathrm{~Hz}), 3.08(\mathrm{~s}, 6 \mathrm{H}), 2.52(\mathrm{~s}, 3 \mathrm{H}) ;{ }^{13} \mathrm{C}$ NMR $(75$ $\left.\mathrm{MHz}, \mathrm{CDCl}_{3}\right) \delta 165.4,153.0,152.7,138.8,130.0,129.3$, $128.8,122.8,120.3,111.2,40.1,23.4 ; \mathrm{MS} m / z 290(\mathrm{M}+\mathrm{H})^{+}$.

6-Chloro- $N$-(2-chloropyridin-3-yl)-4-dimethylaminobenzamide (4d). Yield 94\%; ivory solid; ${ }^{1} \mathrm{H}$ NMR $(300$ $\left.\mathrm{MHz}_{3} \mathrm{CDCl}_{3}\right) \delta 8.94(\mathrm{~d}, 1 \mathrm{H}, J=8.6 \mathrm{~Hz}$ ), 8.26 (br s, $1 \mathrm{H}$ ), $7.80(\mathrm{~d}, 2 \mathrm{H}, J=8.9 \mathrm{~Hz}), 7.31(\mathrm{~d}, 1 \mathrm{H}, J=8.6 \mathrm{~Hz}), 6.73(\mathrm{~d}$, $2 \mathrm{H}, J=8.9 \mathrm{~Hz}), 3.09(\mathrm{~s}, 6 \mathrm{H}) ;{ }^{13} \mathrm{C} \mathrm{NMR}\left(75 \mathrm{MHz}, \mathrm{CDCl}_{3}\right) \delta$ $165.4,153.2,142.6,138.0,131.9,130.9,128.9,123.8$, $119.6,111.2,40.1 ; \mathrm{MS} m / z 310(\mathrm{M}+\mathrm{H})^{+}$.

5-Methyl- $N$-(2-chloropyridin-3-yl)-4-dimethylaminobenzamide (4e). Yield 53\%; white solid; ${ }^{1} \mathrm{H}$ NMR $(300$ $\left.\mathrm{MHz}, \mathrm{CDCl}_{3}\right) \delta 8.78(\mathrm{~d}, 1 \mathrm{H}, J=1.9 \mathrm{~Hz}), 8.29$ (br s, $1 \mathrm{H}$ ), 7.93 (d, $1 \mathrm{H}, J=1.9 \mathrm{~Hz}), 7.82(\mathrm{~d}, 2 \mathrm{H}, J=9.0 \mathrm{~Hz}), 6.74$ (d, $2 \mathrm{H}, J=9.0 \mathrm{~Hz}), 3.09(\mathrm{~s}, 6 \mathrm{H}), 2.53(\mathrm{~s}, 3 \mathrm{H}) ;{ }^{13} \mathrm{C} \mathrm{NMR}(75$ $\left.\mathrm{MHz}_{3} \mathrm{CDCl}_{3}\right) \delta 165.5,153.0,143.4,137.1,133.7,131.9$, $129.1,128.8,120.1,111.2,40.1,18.1 ; \mathrm{MS} m / z 290(\mathrm{M}+\mathrm{H})^{+}$.

5-Chloro- $N$-(2-chloropyridin-3-yl)-4-dimethylaminobenzamide (4f). Yield 79\%; ivory solid; ${ }^{1} \mathrm{H}$ NMR $(300$ $\left.\mathrm{MHz}, \mathrm{CDCl}_{3}\right) \delta 9.04(\mathrm{~d}, 1 \mathrm{H}, J=2.4 \mathrm{~Hz}), 8.31$ (br s, $1 \mathrm{H}$ ), $8.06(\mathrm{~d}, 1 \mathrm{H}, J=2.4 \mathrm{~Hz}), 7.80(\mathrm{~d}, 2 \mathrm{H}, J=9.0 \mathrm{~Hz}), 6.73(\mathrm{~d}$, $2 \mathrm{H}, J=9.0 \mathrm{~Hz}), 3.09(\mathrm{~s}, 6 \mathrm{H}) ;{ }^{13} \mathrm{C} \operatorname{NMR}\left(75 \mathrm{MHz}_{2} \mathrm{CDCl}_{3}\right) \delta$ $165.3,153.2,141.2,137.3,133.1,131.6,128.9,127.8$ $119.4,111.2,40.0 ; \mathrm{MS} m / z 310(\mathrm{M}+\mathrm{H})^{+}$.

General procedure for the synthesis of 2-(4-dimethylaminophenyl)thiazolo[5,4-b]pyridines $1 \mathrm{a}, \mathrm{b}, \mathrm{d}-\mathrm{g}$. To a solution of the appropriate benzamide compound $4(0.5$ mmol) in chlorobenzene $(3-5 \mathrm{~mL})$ was added Lawesson's reagent $(0.3 \mathrm{mmol})$, and the reaction mixture was refluxed for $3 \mathrm{~h}$. After cooling, the reaction mixture was neutralized with $1 \mathrm{~N}$ sodium hydroxide and treated with dichloromethane and water. The organic layer was dried over anhydrous $\mathrm{Na}_{2} \mathrm{SO}_{4}$ and concentrated in vacuo. The residue 
was purified by silica gel column chromatography to give the title compound.

2-(4-Dimethylaminophenyl)thiazolo[5,4-b]pyridine (1a). Yield $35 \%$; brown solid; ${ }^{1} \mathrm{H}$ NMR $\left(300 \mathrm{MHz}_{3} \mathrm{CDCl}_{3}\right) \delta 8.48$ (dd, $1 \mathrm{H}, J=1.5,4.7 \mathrm{~Hz}$ ), 8.18 (dd, $1 \mathrm{H}, J=1.5,8.2 \mathrm{~Hz}$ ), 7.98 (d, $2 \mathrm{H}, J=9.0 \mathrm{~Hz}$ ), 7.38 (dd, $1 \mathrm{H}, J=4.7,8.2 \mathrm{~Hz}), 6.77$ (d, $2 \mathrm{H}, J=9.0 \mathrm{~Hz}), 3.09(\mathrm{~s}, 6 \mathrm{H}) ;{ }^{13} \mathrm{C} \mathrm{NMR}\left(75 \mathrm{MHz}, \mathrm{CDCl}_{3}\right) \delta$ $169.2,158.4,152.5,147.7,145.7,129.0,128.6,121.1$, 111.6, 40.1.

5-Methoxy-2-(4-dimethylaminophenyl)thiazolo[5,4- $b]$ pyridine (1b). Yield $42 \%$; yellow solid; mp $202.0-203.0^{\circ} \mathrm{C}$; IR (KBr): $3431,1718,1495,1360,1287,1262,1178,666$ $\mathrm{cm}^{-1}$; 'H NMR $\left(300 \mathrm{MHz}, \mathrm{CDCl}_{3}\right) \delta 8.41(\mathrm{~d}, 1 \mathrm{H}, J=2.1$ $\mathrm{Hz}), 8.12$ (d, 1H, $J=2.1 \mathrm{~Hz}$ ), 7.94 (d, $2 \mathrm{H}, J=8.9 \mathrm{~Hz}), 6.74$ (d, $2 \mathrm{H}, J=8.9 \mathrm{~Hz}), 3.95(\mathrm{~s}, 3 \mathrm{H}), 3.09(\mathrm{~s}, 6 \mathrm{H}) ;{ }^{13} \mathrm{C} \mathrm{NMR}(75$ $\left.\mathrm{MHz}, \mathrm{CDCl}_{3}\right) \delta 171.3,156.0,152.7,148.2,144.4,129.7$, $129.2,128.0,120.8,111.8,40.2 ; \mathrm{MS} m / z 286(\mathrm{M}+\mathrm{H})^{+}$.

5-Methyl-2-(4-dimethylaminophenyl)thiazolo[5,4-b]pyridine (1d). Yield 46\%; yellow solid; ${ }^{1} \mathrm{H}$ NMR (300 $\left.\mathrm{MHz}, \mathrm{CDCl}_{3}\right) \delta 8.06(\mathrm{~d}, 1 \mathrm{H}, J=8.3 \mathrm{~Hz}), 7.96(\mathrm{~d}, 2 \mathrm{H}, J=9.0$ $\mathrm{Hz}), 7.23(\mathrm{~d}, 1 \mathrm{H}, J=8.3 \mathrm{~Hz}), 6.76(\mathrm{~d}, 2 \mathrm{H}, J=9.0 \mathrm{~Hz}), 3.08$ $(\mathrm{s}, 6 \mathrm{H}), 2.68(\mathrm{~s}, 3 \mathrm{H}) ;{ }^{13} \mathrm{C} \mathrm{NMR}\left(75 \mathrm{MHz}, \mathrm{CDCl}_{3}\right) \delta 168.1$, $157.7,155.1,152.4,145.6,128.8,121.3,121.2,111.6,40.2$, 24.3; MS $m / z 270(\mathrm{M}+\mathrm{H})^{+}$.

5-Chloro-2-(4-dimethylaminophenyl)thiazolo[5,4-b]pyridine (1e). Yield 86\%; yellow solid; mp 243.0-244.0 ${ }^{\circ} \mathrm{C}$; IR (KBr): $3436,1604,1471,1426,1372,1189,812 \mathrm{~cm}^{-1}$; ' $\mathrm{H}$ $\operatorname{NMR}\left(300 \mathrm{MHz}, \mathrm{CDCl}_{3}\right) \delta 8.08(\mathrm{~d}, 1 \mathrm{H}, J=8.5 \mathrm{~Hz}), 7.93(\mathrm{~d}$, $2 \mathrm{H}, J=8.9 \mathrm{~Hz}), 7.36(\mathrm{~d}, 1 \mathrm{H}, J=8.5 \mathrm{~Hz}), 6.75(\mathrm{~d}, 2 \mathrm{H}, J=8.9$ $\mathrm{Hz}), 3.08(\mathrm{~s}, 6 \mathrm{H})$; ${ }^{13} \mathrm{C} \mathrm{NMR}\left(75 \mathrm{MHz}, \mathrm{CDCl}_{3}\right) \delta 169.6$, $157.4,152.5,146.6(2 \mathrm{C}), 130.7,129.0,121.8,120.8,40.2$; MS $m / z 290(\mathrm{M}+\mathrm{H})^{+}$.

6-Methyl-2-(4-dimethylaminophenyl)thiazolo[5,4-b]pyridine (1f). Yield 71\%; yellow solid; 'H NMR (300 MHz, $\left.\mathrm{CDCl}_{3}\right) \delta 8.31(\mathrm{~s}, 1 \mathrm{H}), 7.96(\mathrm{~s}, 1 \mathrm{H}), 7.95(\mathrm{~d}, 2 \mathrm{H}, J=8.6 \mathrm{~Hz})$, $6.75(\mathrm{~d}, 2 \mathrm{H}, J=8.6 \mathrm{~Hz}), 3.09(\mathrm{~s}, 6 \mathrm{H}), 2.68(\mathrm{~s}, 3 \mathrm{H}) ;{ }^{13} \mathrm{C} \mathrm{NMR}$ $\left(75 \mathrm{MHz}_{2} \mathrm{CDCl}_{3}\right) \delta 169.5,154.8,152.5,147.7,146.4,131.2$, $129.2,129.0,121.1,111.7,40.2,18.5$; MS $m / z 270(\mathrm{M}+\mathrm{H})^{+}$.

6-Chloro-2-(4-dimethylaminophenyl)thiazolo[5,4-b]pyridine (1g). Yield 52\%; yellow solid; 'H NMR $(300$ $\left.\mathrm{MHz}, \mathrm{CDCl}_{3}\right) \delta 8.41(\mathrm{~d}, 1 \mathrm{H}, J=2.1 \mathrm{~Hz}), 8.12(\mathrm{~d}, 1 \mathrm{H}, J=2.1$ $\mathrm{Hz}$ ), 7.94 (d, $2 \mathrm{H}, J=8.9 \mathrm{~Hz}), 6.74$ (d, $2 \mathrm{H}, J=8.9 \mathrm{~Hz}), 3.09$ $(\mathrm{s}, 6 \mathrm{H}) ;{ }^{3} \mathrm{C} \mathrm{NMR}\left(75 \mathrm{MHz}, \mathrm{CDCl}_{3}\right) \delta 171.3,156.0,152.7$, $148.2,144.4,129.7,129.2,128.0,120.8,111.8,40.2$; MS $m / z 286(\mathrm{M}+\mathrm{H})^{+}$.

5-Hydroxy-2-(4-dimethylaminophenyl)thiazolo[5,4-b]pyridine (1c). To a solution of $1 \mathbf{b}(29 \mathrm{mg}, 0.1 \mathrm{mmol})$ in dichloromethane $(2 \mathrm{~mL})$ was added tribromoborane $(1 \mathrm{~mL}$, $1 \mathrm{M} \mathrm{BBr}_{3}$ in dichloromethane, $1.0 \mathrm{mmol}$ ) at $0^{\circ} \mathrm{C}$, and the reaction mixture was refluxed for $12 \mathrm{~h}$. After cooling, the reaction mixture was neutralized with $1 \mathrm{~N}$ sodium hydroxide and treated with dichloromethane and water. The organic layer was dried over anhydrous $\mathrm{Na}_{2} \mathrm{SO}_{4}$ and concentrated in vacuo. The residue was purified by silica gel column chromatography to give $1 \mathrm{c}(10 \mathrm{mg}, 37 \%)$ : yellow solid; ' $\mathrm{H}$ $\operatorname{NMR}\left(300 \mathrm{MHz}, \mathrm{CDCl}_{3}\right) \delta 11.41(\mathrm{~s}, 1 \mathrm{H}), 8.06(\mathrm{~d}, 1 \mathrm{H}, J=$ $8.8 \mathrm{~Hz}), 7.77(\mathrm{~d}, 2 \mathrm{H}, J=8.7 \mathrm{~Hz}), 7.23(\mathrm{~d}, 1 \mathrm{H}, J=8.8 \mathrm{~Hz})$, $6.77(\mathrm{~d}, 2 \mathrm{H}, J=8.7 \mathrm{~Hz}), 3.08(\mathrm{~s}, 6 \mathrm{H}) ;{ }^{13} \mathrm{C} \mathrm{NMR}(75 \mathrm{MHz}$, $\left.\mathrm{CDCl}_{3}\right) \delta 171.5,156.2,153.0,148.3,144.9,130.3,129.7$, $128.4,121.0,111.9$.

General procedure for the synthesis of 2-(4-dimethylaminophenyl)oxazolo[5,4-b]pyridines 11-0. To a solution of diphosphorus pentoxide (439 $\mathrm{mg}, 1.5 \mathrm{mmol}$ ) and hexamethyldisiloxane $(1.3 \mathrm{~mL}, 6.0 \mathrm{mmol})$ in 1,2-dichlorobenzene $(3 \mathrm{~mL})$ was added the appropriate benzamide compound $4(0.5 \mathrm{mmol})$ at $140^{\circ} \mathrm{C}$, and the reaction mixture was stirred for $2 \mathrm{~d}$. When the reaction was completed, the reaction mixture was treated with $10 \% \mathrm{NaHCO}_{3}$. The mixture was extracted with ethyl acetate, dried over anhydrous $\mathrm{Na}_{2} \mathrm{SO}_{4}$, and concentrated in vacuo. The residue was purified by silica gel column chromatography to give the title compound (for $11, \mathrm{~m})$. Polyphosphoric acid $(2.0 \mathrm{~g})$ was added to the appropriate benzamide compound $4(0.5 \mathrm{mmol})$ at $140^{\circ} \mathrm{C}$, and the reaction mixture was stirred for $1 \mathrm{~d}$. When the reaction was completed, the reaction mixture was neutralized with $1 \mathrm{~N} \mathrm{NaOH}$. The mixture was extracted with ethyl acetate, dried over anhydrous $\mathrm{Na}_{2} \mathrm{SO}_{4}$, and concentrated in vacuo. The residue was purified by silica gel column chromatography to give the title compound (for $\mathbf{1 n}, \mathbf{0}$ ).

2-(4-Dimethylaminophenyl)oxazolo[5,4-b]pyridine (11). Yield $26 \%$; pale yellow solid; 'H NMR ( $\left.300 \mathrm{MHz}, \mathrm{CDCl}_{3}\right) \delta$ $8.25(\mathrm{dd}, 1 \mathrm{H}, J=1.4,5.0 \mathrm{~Hz}), 8.15(\mathrm{~d}, 2 \mathrm{H}, J=9.0 \mathrm{~Hz}), 7.96$ (dd, $1 \mathrm{H}, J=1.4,7.8 \mathrm{~Hz}), 7.15(\mathrm{dd}, 1 \mathrm{H}, J=5.0,7.8 \mathrm{~Hz}), 6.79$ (d, $2 \mathrm{H}, J=9.0 \mathrm{~Hz}), 3.10(\mathrm{~s}, 6 \mathrm{H}) ;{ }^{13} \mathrm{C}$ NMR $(75 \mathrm{MHz}$, $\left.\mathrm{CDCl}_{3}\right) \delta 164.1,159.8,152.6,143.2,134.4,129.5,126.9$, $120.7,113.8,112.1,40.4 ; \mathrm{MS} m / z 240(\mathrm{M}+\mathrm{H})^{+}$.

5-Methyl-2-(4-dimethylaminophenyl)oxazolo[5,4-b]pyridine (1m). Yield $11 \%$; pale yellow solid; ${ }^{1} \mathrm{H}$ NMR $(300$ $\left.\mathrm{MHz}_{3} \mathrm{CDCl}_{3}\right) \delta 8.11(\mathrm{~d}, 2 \mathrm{H}, J=9.0 \mathrm{~Hz}), 7.83(\mathrm{~d}, 1 \mathrm{H}, J=7.9$ $\mathrm{Hz}), 7.13(\mathrm{~d}, 1 \mathrm{H}, J=7.9 \mathrm{~Hz}), 6.78(\mathrm{~d}, 2 \mathrm{H}, J=9.0 \mathrm{~Hz}), 3.11$ $(\mathrm{s}, 6 \mathrm{H}), 2.67(\mathrm{~s}, 3 \mathrm{H}) ;{ }^{13} \mathrm{C} \mathrm{NMR}\left(75 \mathrm{MHz}, \mathrm{CDCl}_{3}\right) \delta 163.5$, $159.5,152.8,152.7,132.0,129.2,126.9,120.1,113.5,111.6$, $40.1,24.1 ; \mathrm{MS} m / z 254(\mathrm{M}+\mathrm{H})^{+}$.

5-Chloro-2-(4-dimethylaminophenyl)oxazolo[5,4-b]pyridine (1n). Yield 37\%; pale yellow solid; ${ }^{1} \mathrm{H}$ NMR $(300$ $\left.\mathrm{MHz}_{3} \mathrm{CDCl}_{3}\right) \delta 8.10(\mathrm{~d}, 2 \mathrm{H}, J=9.0 \mathrm{~Hz}), 7.89(\mathrm{~d}, 1 \mathrm{H}, J=8.1$ $\mathrm{Hz}), 7.31$ (d, $1 \mathrm{H}, J=8.1 \mathrm{~Hz}), 6.81(\mathrm{~d}, 2 \mathrm{H}, J=9.0 \mathrm{~Hz}), 3.10$ $(\mathrm{s}, 6 \mathrm{H}) ;{ }^{13} \mathrm{C}$ NMR $\left(75 \mathrm{MHz} \mathrm{CDCl}_{3}\right) \delta 164.6,158.6,152.8$, $144.4,133.6,129.5,128.7,120.9,113.1,111.9,40.3$; MS $m / z 274(\mathrm{M}+\mathrm{H})^{+}$.

6-Chloro-2-(4-dimethylaminophenyl)oxazolo[5,4-b]pyridine (10). Yield 53\%; pale yellow solid; ${ }^{1} \mathrm{H}$ NMR $(300$ $\left.\mathrm{MHz}_{3} \mathrm{CDCl}_{3}\right) \delta 8.27(\mathrm{~s}, 1 \mathrm{H}), 8.00(\mathrm{~d}, 2 \mathrm{H}, J=9.0 \mathrm{~Hz}), 7.54$ $(\mathrm{s}, 1 \mathrm{H}), 6.86(\mathrm{~d}, 2 \mathrm{H}, J=9.0 \mathrm{~Hz}), 3.11(\mathrm{~s}, 6 \mathrm{H}) ;{ }^{13} \mathrm{C} \mathrm{NMR}(75$ $\left.\mathrm{MHz}_{3} \mathrm{CDCl}_{3}\right) \delta 165.5,158.4,153.6,141.5,135.7,129.7$, $128.0,127.1,112.2,111.8,40.4$; MS $m / z 278(\mathrm{M}+\mathrm{H})^{+}$.

General procedure for the synthesis of $N$-(2-chloropyridin-3-yl)-4-nitrobenzamides $\mathbf{5 a}, \mathbf{b}$. To a solution of the appropriate 3 -aminopyridine compound $3(10.0 \mathrm{mmol})$ in pyridine $(25 \mathrm{~mL})$ was added 4-nitrobenzoyl chloride $(10.0$ mmol) in pyridine $(5 \mathrm{~mL})$, and the reaction mixture was stirred at room temperature for $18 \mathrm{~h}$. When the reaction was completed, the reaction mixture was treated with water. The resulting precipitate was collected by filtration to give the 
compound 5.

N-(2-Chloropyridin-3-yl)-4-nitrobenzamide (5a). Yield $90 \%$; ivory solid; ${ }^{1} \mathrm{H}$ NMR $\left(300 \mathrm{MHz}, \mathrm{CDCl}_{3}\right) \delta 8.88$ (dd, $1 \mathrm{H}, J=1.7,8.2 \mathrm{~Hz}$ ), 8.44 (br s, $1 \mathrm{H}), 8.40$ (d, $2 \mathrm{H}, J=8.8 \mathrm{~Hz}$ ), 8.21 (dd, $1 \mathrm{H}, J=1.7,4.7 \mathrm{~Hz}), 8.10$ (d, $2 \mathrm{H}, J=8.8 \mathrm{~Hz}), 7.36$ (dd, $1 \mathrm{H}, J=4.7,8.2 \mathrm{~Hz}) ;{ }^{13} \mathrm{C}$ NMR $\left(75 \mathrm{MHz}, \mathrm{CDCl}_{3}\right) \delta$ $163.6,150.2,144.7,140.4,139.3,131.5,129.2,128.4$, 124.3, 123.6; MS $m / z 228(\mathrm{M}+\mathrm{H})^{+}$

6-Methoxy- $N$-(2-chloropyridin-3-yl)-4-nitrobenzamide (5b). Yield 52\%; ivory solid; ${ }^{1} \mathrm{H} \mathrm{NMR}\left(300 \mathrm{MHz}, \mathrm{CDCl}_{3}\right) \delta$ 8.64 (d, $1 \mathrm{H}, J=8.8 \mathrm{~Hz}$ ), 8.38 (d, $2 \mathrm{H}, J=8.7 \mathrm{~Hz}), 8.14$ (br s, $1 \mathrm{H}), 8.09$ (d, $2 \mathrm{H}, J=8.7 \mathrm{~Hz}), 6.79(\mathrm{~d}, 1 \mathrm{H}, J=8.8 \mathrm{~Hz}), 3.95$ $(\mathrm{s}, 3 \mathrm{H}) ;{ }^{13} \mathrm{C} \mathrm{NMR}\left(75 \mathrm{MHz}, \mathrm{CDCl}_{3}\right) \delta 163.3,160.0,150.0$, $139.6,137.4,133.7,128.3,124.7,110.1,54.3$; MS $m / z 272$ $(\mathrm{M}+\mathrm{H})^{+}$.

General procedure for the synthesis of 2-(4-nitrophenyl)thiazolo[5,4-b]pyridines $6 a, b$. To a solution of the appropriate benzamide compound $5(2.0 \mathrm{mmol})$ in chlorobenzene (12-15 $\mathrm{mL}$ ) was added Lawesson's reagent $(1.2$ mmol), and the reaction mixture was refluxed for $3 \mathrm{~h}$. After cooling, the reaction mixture was neutralized with $1 \mathrm{~N}$ sodium hydroxide and treated with dichloromethane and water. The organic layer was dried over anhydrous $\mathrm{Na}_{2} \mathrm{SO}_{4}$ and concentrated in vacto. The residue was purified by silica gel column chromatography to give the compound 6 .

2-(4-Nitrophenyl)thiazolo[5,4-b]pyridine (6a). Yield $58 \%$; pale yellow solid; ${ }^{1} \mathrm{H}$ NMR $\left(300 \mathrm{MHz}, \mathrm{CDCl}_{3}\right) \delta 8.65$ (dd, $1 \mathrm{H}, J=1.5,4.6 \mathrm{~Hz}), 8.37(\mathrm{~m}, 3 \mathrm{H}), 8.30$ (d, $2 \mathrm{H}, J=8.9$ $\mathrm{Hz}), 7.52$ (dd, $1 \mathrm{H}, J=4.6,8.2 \mathrm{~Hz}$ ); ${ }^{13} \mathrm{C}$ NMR $(75 \mathrm{MHz}$, $\left.\mathrm{CDCl}_{3}\right) \delta 165.5,158.6,149.4,148.1,147.2,138.9,130.9$, $128.4,124.4,122.0 ; \mathrm{MS} m / z 258(\mathrm{M}+\mathrm{H})^{+}$.

5-Methoxy-2-(4-nitrophenyl)thiazolo[5,4-b]pyridine (6b). Yield $80 \%$; yellow solid; ${ }^{1} \mathrm{H}$ NMR $\left(300 \mathrm{MHz}, \mathrm{CDCl}_{3}\right) \delta$ $8.35(\mathrm{~d}, 2 \mathrm{H}, J=9.0 \mathrm{~Hz}), 8.20(\mathrm{~m}, 3 \mathrm{H}), 6.93(\mathrm{~d}, 1 \mathrm{H}, J=8.8$ $\mathrm{Hz}), 3.95(\mathrm{~s}, 3 \mathrm{H}) ;{ }^{13} \mathrm{C} \mathrm{NMR}\left(75 \mathrm{MHz}, \mathrm{CDCl}_{3}\right) \delta 162.9$, $161.2,148.8,142.8,139.4,133.2,127.7,124.4,111.4,54.3$.

General procedure for the synthesis of 2-(4-aminophenyl)thiazolo[5,4-b]pyridines $1 \mathrm{~h}, \mathbf{i}$. To a solution of the appropriate nitrophenyl compound $6(1.0 \mathrm{mmol})$ in ethanol $(10 \mathrm{~mL})$ was added tin chloride $(5.0 \mathrm{mmol})$, and the reaction mixture was refluxed for $6-18 \mathrm{~h}$. When the reaction was completed, the reaction mixture was treated with $10 \%$ $\mathrm{NaHCO}_{3}$. The mixture was extracted with ethyl acetate, dried over anhydrous $\mathrm{Na}_{2} \mathrm{SO}_{4}$, and concentrated in vacuo. The residue was purified by silica gel column chromatography to give the title compound.

2-(4-Aminophenyl)thiazolo[5,4-b]pyridine (1h). Yield $>99 \%$; yellow solid; mp 236.5-238.0 ' C; IR (KBr): 3320 , $3201,1604,1471,1439,1296,1217,1177 \mathrm{~cm}^{-1}$; 'H NMR $\left(300 \mathrm{MHz}, \mathrm{CDCl}_{3}\right) \delta 8.49$ (dd, $\left.1 \mathrm{H}, J=1.3,4.7 \mathrm{~Hz}\right), 8.18$ (dd, $1 \mathrm{H}, J=1.3,8.2 \mathrm{~Hz}), 7.90(\mathrm{~d}, 2 \mathrm{H}, J=8.5 \mathrm{~Hz}), 7.38(\mathrm{dd}, 1 \mathrm{H}, J$ $=4.7,8.2 \mathrm{~Hz}), 6.75(\mathrm{~d}, 2 \mathrm{H}, J=8.5 \mathrm{~Hz}), 4.11$ (br s, $2 \mathrm{H}) ;{ }^{13} \mathrm{C}$ $\mathrm{NMR}\left(75 \mathrm{MHz}, \mathrm{CDCl}_{3}\right) \delta 169.0,158.3,149.8,147.5,146.1$, $129.3,129.0,123.7,121.2,114.8 ; \mathrm{MS} m / z 228(\mathrm{M}+\mathrm{H})^{+}$.

5-Methoxy-2-(4-aminophenyl)thiazolo[5,4-b]pyridine (1i). Yield $88 \%$; yellow solid; ${ }^{1} \mathrm{H} \mathrm{NMR}\left(300 \mathrm{MHz}, \mathrm{CDCl}_{3}\right) \delta$ 8.06 (d, $1 \mathrm{H}, J=8.8 \mathrm{~Hz}), 7.84$ (d, $2 \mathrm{H}, J=8.6 \mathrm{~Hz}$ ), 6.82 (d,
$1 \mathrm{H}, J=8.8 \mathrm{~Hz}), 6.74(\mathrm{~d}, 2 \mathrm{H}, J=8.6 \mathrm{~Hz}), 4.40(\mathrm{~s}, 3 \mathrm{H}), 4.12$ (br s, $2 \mathrm{H}) ;{ }^{13} \mathrm{C} \mathrm{NMR}\left(75 \mathrm{MHz}, \mathrm{CDCl}_{3}\right) \delta 165.2,161.9$, $154.8,149.1,142.7,131.9,128.7,124.2,114.8,109.8,54.1$.

General procedure for the synthesis of 2-(4-methylaminophenyl)thiazolo $[5,4-b]$ pyridines $1 \mathrm{j}$, k. To a solution of the appropriate aminophenyl compound $6(0.2 \mathrm{mmol})$ and paraformaldehyde $(0.3-0.8 \mathrm{mmol})$ in methanol $(3 \mathrm{~mL})$ was added $25 \mathrm{wt} \% \mathrm{NaOMe}$ in methanol $(0.6-0.8 \mathrm{mmol})$ at $0^{\circ} \mathrm{C}$. After being stirred at reflux for $2 \mathrm{~h}$, sodium borohydride $(0.6-0.8 \mathrm{mmol})$ was slowly added and the reaction mixture was further refluxed for $3 \mathrm{~h}$. When the reaction was completed, the reaction mixture was extracted with ethyl acetate, dried over anhydrous $\mathrm{Na}_{2} \mathrm{SO}_{4}$, and concentrated in vacto. The residue was purified by silica gel column chromatography to give the title compound.

2-(4-Methylaminophenyl)thiazolo[5,4-b]pyridine (1j). Yield $73 \%$; yellow solid; 'H NMR $\left(300 \mathrm{MHz}_{3} \mathrm{CDCl}_{3}\right) \delta$ 8.48 (dd, $1 \mathrm{H}, J=1.4,4.7 \mathrm{~Hz}$ ), 8.17 (dd, $1 \mathrm{H}, J=1.4,8.2 \mathrm{~Hz}$ ), 7.94 (d, $2 \mathrm{H}, J=8.8 \mathrm{~Hz}$ ), 7.37 (dd, $1 \mathrm{H}, J=4.7,8.2 \mathrm{~Hz}$ ), 6.67 (d, $2 \mathrm{H}, J=8.8 \mathrm{~Hz}$ ), 4.21 (br s, $1 \mathrm{H}), 2.94\left(\mathrm{~s}, 3 \mathrm{H}\right.$ ); ${ }^{13} \mathrm{C} \mathrm{NMR}$ $\left(75 \mathrm{MHz}, \mathrm{CDCl}_{3}\right) \delta 169.2,158.3,152.0,147.6,145.9,129.3$, $128.7,122.3,121.2,112.0,30.3$.

5-Methoxy-2-(4-methylaminophenyl)thiazolo[5,4-b]pyridine (1k). Yield $74 \%$; yellow solid; mp $227.0-228.0^{\circ} \mathrm{C}$; IR (KBr): $3306,1608,1463,1373,1265,1181 \mathrm{~cm}^{-1}$; 'H NMR $\left(300 \mathrm{MHz}, \mathrm{CDCl}_{3}\right) \delta 8.05(\mathrm{~d}, 1 \mathrm{H}, J=8.8 \mathrm{~Hz}), 7.86(\mathrm{~d}, 2 \mathrm{H}, J$ $=8.6 \mathrm{~Hz}), 6.81(\mathrm{~d}, 1 \mathrm{H}, J=8.8 \mathrm{~Hz}), 6.65(\mathrm{~d}, 2 \mathrm{H}, J=8.6 \mathrm{~Hz})$, 4.11 (br s, $1 \mathrm{H}), 4.01$ (s, 3H), $2.91(\mathrm{~s}, 3 \mathrm{H}) ;{ }^{13} \mathrm{C}$ NMR $(75$ $\left.\mathrm{MHz}, \mathrm{CDCl}_{3}\right) \delta 165.5,161.8,154.8,151.4,142.8,131.7$, $128.6,122.8,112.1,109.7,54.1,30.3 ; \mathrm{MS} m / 2272(\mathrm{M}+\mathrm{H})^{+}$.

Acknowledgments. We are grateful to the Ministry of Science and Technology (MOST) and Ministry of Commerce, Industry and Energy (MCIE) of Korea for financial support.

\section{References}

I. Förstl, H.; Kurz, A. Etr. Arch. Psychiatry Clin. Neurosci. 1999, $240,288$.

2. Masters, C. L.; Simms, G.; Weinman, N. A.; Multhaup, G.; McDonald, B. L.; Beyreuther, K. Proc. Natl. Acad. Sci. Li.S.A. $1985,82,4245$.

3. Hardy, J.; Selkoe, D. J. Science 2002, 207, 353.

4. Gandy, S. J. Clin. Invest. 2005, $/ 15,1121$.

5. Lomakin, A.; Teplow, D. B.; Kirschner, D. A.; Benedek. G B. Proc. Natl. Acad. Sci. U.S.A. 1996, 93, 1125.

6. Zerovnik, E. Em: J. Biochem. 2002, 269, 3362.

7. Corder, E. H.; Saunders, A. M.; Stritumauter, W. J.; Schmechel, D. E.; Gaskell, P. C.; Small, G. W.; Roses, A. D.; Haines, J. L.; Paticak-Vance, M. A. Science 1993, 261,921.

8. Tanzi, R. E.; Bertram, L. Neuron 2001, 32, 184.

9. Jarreul, J. T.; Berger, E. P.; Lansbury, P. T. Biochemistry 1993, \$2, 4693.

10. Hammarstrom, P; Wiseman, R. L.; Powers, E. T, Kelly, J. W. Science 2003, 200, 713.

I1. Vickers, J. C.; Dickson, T. C.; Adlard, P. A.; Sounders, H. L.; King. C. E.; McCormack, G. Prog. Neurobiol. 2000, 60, 139.

12. Thorset, E. D.; Latimer, L. H. Curr, Opin, Chem. Biol. 2000, 4, 377 .

13. Skovronsky, D. M.; Lee, V. M. Trends Pharmacol. Sci. 2000, 21, 
161.

14. Lee, C. W.; Zhuang, Z. P.; Kung, M. P.; Plössl, K.; Skovronsky, D.; Gur, T.; Hou, C.; Trojanowski, J. Q.; Lee, V. M. Y.; Kung, H. F. J. Med. Chent. 2001, 44, 2270.

15. Mauhis, C. A.; Wang, Y,; Klunk, W. E. Cum: Phorm. Design 2004, 10,1469

16. Lim, G. P.; Chu, T.; Yang, F.; Beech, W.; Frautschy, S. A.; Cole, G M. J. Neurosci. 2001, 21, 8370.

17. Yang, F.; Lim, G. P.; Begum, A. N.; Ubeda, O. J.; Simmons, M. R.; Ambegaokar, S. S.; Chen, P. P.; Kayed, R.; Glabe, C. G.; Frautschy, S. A.; Cole, G. M. J. Biol. Chem. 2005, 280, 5892.

18. Ono, M.; Wilson, A.; Nobrega, J.; Westaway, D.; Verhoeff, N. P.; Zhuang, Z.-P.; Kung, M.-P.; Kung, H. F. Nucl. Med. Biol. 2003 , 30,565 .

19. Howlett, D. R.; Perry, A. E.; Godfrey, F.; Swatton, J. E.; Jennings, K. H.; Spitzfaden, C.; Wadsworth, H.; Wood, S. J.; Markwell, R.
E. Biochem. J. 1999, 340, 283.

20. Mathis, C. A.; Wang, Y.; Holt, D. P.; Huang, G.-f.; Debnath, M. L.; Klunk. W. E. J. Med. Chem. 2003, 46, 2740.

2l. Klunk, W. E.; Engler, H.; Nordberg, A.; Wang, Y.; Blomqvist, G.; Hol, D. P.; Bergsitrom, M.; Savitcheva, I.; Huan, Gi-f.; Estrada, S.; Ausen, B.; Debnath, M. L.; Barletta, J.; Price, J. C.; Sndell, J.; Lopresti, B. J.; Wall, A.; Koivisto, P.; Antoni, G.; Mathis, C. A.; Langstrom, B. Ann. Netrol. 2004, 55, 306.

22. Jouve, K.; Bergman, J. J. Heterocycl. Chem. 2003, 40, 261.

23. Couture, A.; Grandclaudon, P. Hetrocycles 1984, 22, 1383.

24. Flouzat, C.; Guillaumet, G. Sywhesis 1990, 64.

25. Hiroya, K.; Suzuki, N.; Yasuhara, A.; Egawa, Y.; Kasano, A.; Sakamoto, T. J. Chent. Soc., Perkin Trans. I 2000, 4339.

26. Barluenga, J.; Bayon, A. M.; Asensio, G. J. Chem. Soc. Chent. Conminin. 1984. 1334.

27. Revine III, H. Arch, Biochem. Biophys, 2002, 404, 106. 